\title{
Compressed Sensing based Signal and Data Acquisition in Wireless Sensor Networks and Internet of Things
}

\author{
Shancang Li, Li Da Xu, and Xinheng Wang
}

\begin{abstract}
The emerging compressed sensing (CS) theory can significantly reduce the number of sampling points that directly corresponds to the volume of data collected, which means that part of the redundant data is never acquired. It makes it possible to create stand-alone and net-centric applications with fewer resources required in Internet of things (IoT). CS-based signal and information acquisition/compression paradigm combines the nonlinear reconstruction algorithm and random sampling on a sparse basis that provides a promising approach to compress signal and data in information systems. This paper investigates how CS can provide new insights into data sampling and acquisition in wireless sensor networks and IoT. At first, we briefly introduce the CS theory in respect of the sampling and transmission coordination during the network lifetime through providing a compressed sampling process with low computation costs. Then, a compressed sensing-based framework is proposed for IoT, in which the end nodes measure, transmit, and store the sampled data in the framework. Then, an efficient cluster-sparse reconstruction algorithm is proposed for in-network compression aiming at more accurate data reconstruction and lower energy efficiency. Performance is evaluated with respect to network size using datasets acquired by a real-life deployment.
\end{abstract}

Index Terms-Compressed Sensing, Wireless Sensor Networks, Industrial Informatics, Internet of Things, Information Systems, Enterprise Systems

\section{INTRODUCTION}

Researchers found that in information systems, wireless sensor networks (WSNs), and Internet of Things (IoT), many types of information has a property called sparseness in transformation process which allows certain number of samples enabling capturing all required information without loss of information [1], [2], [3], [4]. IoT has been emerged as a technological revolution in the information industry [1], [2]. IoT is expected to be a world-wide network of interconnected objects, and its development depends on a number of new technologies, such as WSNs, cloud computing, and information sensing [2], [3], [4]. In IoT-based information systems, a low-cost data acquisition system is necessary to

Manuscript received December 28, 2011;revised October 26, 2012.

Copyright (c) 2009 IEEE. Personal use of this material is permitted. However, permission to use this material for any other purposes must be obtained from the IEEE by sending a request to pubs-permissions@ieee.org.

Shancang $\mathrm{Li}$ is with College of Engineering, Swansea University, Swansea SA2 8PP, UK (Phone: +44-1792-602802, Fax: +44-1792-602802, Email:s.li@swansea.ac.uk).

$\mathrm{Li} \mathrm{Da} \mathrm{Xu}$ is with the Institute of Computing Technology, Chinese Academy of Sciences, Beijing 100190, China; Old Dominion University, Norfolk, VA 23529, USA

Xinheng Wang is with College of Engineering, Swansea University, Swansea SA2 8PP, UK (xinheng.wang@swansea.ac.uk). effectively collect and process the data and information at IoT end nodes [2], [3], [5], [6]. WSNs have the potential of a wide range of applications in many industrial systems. WSNs can be integrated into the IoT, which consists of a number of interconnected sensor nodes [3], [4], [5].

An IoT can involve thousands of independent components including computers, sensors, RFID tags, or mobile phones, all are capable of generating and communicating data, in which many techniques are involved for data collection, transmission, and storage [2], [4], [7]. In IoT, a desirable data compression ratio is very important, which cannot be obtained by current methods without introducing unacceptable distortions [8], [9]. Furthermore, for most data compression solutions in IoT, three main problems must be solved: resolution, sensitivity, and reliability [2], [10], [11], [12].

Recently, an emerging theory named compressed sensing (CS) has been extensively investigated, with which the data or signals can be efficiently sampled and accurately reconstructed with much fewer samples than Nyquist theory [3], [4], [8], [13]. CS relies on the facts that many types of information has a property called sparseness in transformation process. The required information could be obtained from these compressedly sampled signals as well as the whole signals sampled by Nyquist theory [14]. The CS changes the rule of data acquisition game in information systems by exploiting a priori data sparsity information [5]. The applications of CS for data acquisition in WSNs have been studied recently [4], [15], [16], [17]. In [4], Haupt investigated the compressed sensing for networked data in WSNs through considering the distributed data sources and their sampling, transmission, and storage. In [15], Fazel proposed a random access compressed sensing scheme for long-term data gathering in large-scale sensor networks, which is expected to prolong the life-time of a sensor network. In [16], Pudlewski et al. applied the CS theory to the jointly control of the video streaming rates to reduce the communication cost and increase network capacity. In [17], Mamaghanian investigated CS for energy-efficient signals gathering in a wireless body sensor network.

However, for the first time, our work studies information acquisition in IoT and WSNs with CS from the perspective of data compressed sampling, robust transmission, and accurate reconstruction to reduce the energy consumption, computation costs, data redundancy, and increase the network capacity. A common task of an IoT end node is to transmit the sensed data to specific node or fusion center (FC), however, how to efficiently acquire, store, and transmit among a large number 
of source nodes remains a challenge [4], [14]. This paper considers a particular situation that involves with distributed information sources of data and their acquisition, transmission, storage, and processing in a large-scale IoT [18]. The contributions are summarized as follows:

1) We formulate the problem of data acquisition based on compressed sampling in IoT and WSNs. This is the first time to apply compressed sampling scheme in IoT with a theoretical basis.

2) A CS-based information acquisition framework is proposed for IoT, which involves the compressed sampling at IoT end node, information transmission over IoT, and accurate data reconstruction at FC. In this framework, the noise model, communication load, and recovery accuracy are considered for its industrial applications.

3) By taking the correlation of sensing data over IoT and WSNs into consideration, an adaptive sparse representation and corresponding signal reconstruction algorithm are proposed which offer a higher accuracy and lower computational complexity compared with pre-existing group/cluster-sparse reconstruction algorithms.

In the following sections, we will propose a compressed sensing-based data gathering scheme, in which the compressed sensing is able to provide a compressed sampling stage with low computational costs. In Section II, we introduce the main idea of data compression using compressed sensing. In Section III, we propose a flexible data acquisition framework for IoT based on compressed sensing. Experiments of data acquisition and reconstruction are proposed for IoT to demonstrate the effectiveness of the proposed approaches in Section IV. Section $\mathrm{V}$ concludes the paper.

\section{COMPRESSED SENSING}

In a network with $n$ nodes, each node collects or generates data $x_{j}, j=1, \ldots, n$. For simplicity, we assume that each sample $x_{j}$ is a scalar data (such as temperature, pressure, etc.) and the collected data is a vector $\mathbf{x}=\left[x_{1}, \ldots, x_{n}\right]^{T}$, namely measurements. Thesemeasurements are distributed and can be shared over the network. IoT may be very large, and the collection of $\mathbf{x}$ at an IoT node might be inefficient and unreliable. However, compressed sensing theory makes it possible to accurately reconstruct $\mathbf{x}$ based on a highly compressed decentralized measurement of $\mathbf{x}$ [4], [19], [20].

Compressed sensing-based data acquisition is very different from the decentralized data acquisition in IoT networks. Considering a data acquisition model: $\mathbf{y}=\mathbf{\Phi} \mathbf{x}$, in which $\boldsymbol{\Phi}$ denotes an $m$-by- $n$ measurement matrix with $m$ far less than $n$. The measurement $\mathbf{y}$ is an $m \times 1$ vector, with the number of elements being far fewer than that of the original data $\mathbf{x}$. Therefore, $\mathbf{y}$ can be transmitted, processed, and stored with much lower resource requirements than $\mathbf{x}$. In compressed sensing theory, with a properly designed measurement matrix $\boldsymbol{\Phi}$, it is possible to recover $\mathbf{x}$ from $\mathbf{y}$ within a reasonable accuracy whenever $\mathbf{x}$ is compressible.

$$
\mathbf{y}=\boldsymbol{\Phi} \mathbf{x}
$$

Compressed sensing is able to measure the data without requiring any specific prior knowledge [4], [21], [22]. The required data over the whole network can be reconstructed based on the measurements as described in Eq.(1), providing its size $m$ is much smaller than $n$ [23]. In compressed sensing-based WSNs and IoT, two features can be obtained for effective data analysis: (1) The compressed sensing-based method is able to work cooperatively between the nodes, which means that the collected or generated data by each node can be distributively processed even without a fusion centre (FC); (2) The data can be sampled and reconstructed without prior knowledge. These two features make the compressed sensing easier to be used for applications where gathering data is expensive.

\section{A. Conditions for Compressed Sensing}

Definition 1: If a signal $\mathbf{x}=\left[x_{1}, \ldots, x_{n}\right]^{T}$ can be represented over a set of orthonormal $n \times 1$ vector $\left\{\psi_{i}\right\}_{i=1}^{n}$, then it can be said sparse

$$
\mathbf{x}=\sum_{i=1}^{n} \theta_{i} \psi_{i}, \text { or }, \theta=\mathbf{\Psi}^{\mathbf{T}} \mathbf{x}
$$

in which $\theta$ is an $n \times 1$ vector that denotes the weights vector, and $\theta_{i}=<\mathbf{x}, \psi_{i}>; \boldsymbol{\Psi}=\left[\psi_{1}, \ldots, \psi_{n}\right]$ is the basis matrix. If there are $k(k \ll n)$ nonzero coefficients in $\theta$, then the signal can be said as $k$-sparse.

A signal might be compressible if it can be represented in terms of a sparse expansion. In fact, compressible signals are rather ubiquitous which allow compressed sensing in many far-reaching applications such as data acquisition, data compression, network coding, and others [24]. The sparse signal or data is measured by taking a smaller number of samples $(m)$ from the original $\mathbf{x}$ using a linear/convex programming operator $\boldsymbol{\Phi}$, hence Eq.(1) can be rewritten as

$$
\mathbf{y}=\mathbf{\Phi} \mathbf{x}=\boldsymbol{\Phi} \boldsymbol{\Psi} \theta=\mathbf{A} \theta
$$

where $\boldsymbol{\Phi}=\left[\phi_{1}, \phi_{2}, \ldots, \phi_{m}\right]^{T}, \mathbf{A}=\boldsymbol{\Phi} \boldsymbol{\Psi}, k \leq m \ll n$, and the $n \times 1$ vector $\mathbf{x}$ is compressed into an $m \times 1$ measurement vector $\mathbf{y}$. Eq.(3) admits many to an infinite number of solutions. In order to find the sparsest solution, it can be easily solved as an optimization problem by maximizing "measurement of sparsity" while simultaneously satisfying Eq.(3). To find a unique sparse solution of Eq.(3), the measurement matrix needs to be successfully designed. Two main categories of measurement ensembles can be directly applied in compressed sensing [4], [25], [26], [27]:

Random measurements, $\boldsymbol{\Phi}$ is not explicitly used, in which the measurement $\mathbf{y}$ is random linear combinations of the entries of $\mathbf{x}$. The measurement matrix can be Fourier, Binary or Gaussian. In compressed sensing framework, the incoherent measurements can be obtained by random ensembles. Randomness is likely to provide incoherent projections [25], [26].

Incoherent measurements, in which $\boldsymbol{\Phi}$ is deterministic that is assumed to be incoherent with $\boldsymbol{\Psi}$. The incoherence between $\boldsymbol{\Phi}$ and $\boldsymbol{\Psi}$ can be measured by their mutual coherence [26].

Definition 2: The coherence of two vectors $\phi_{i}$ and $\psi_{k}$ can be defined as

$$
\mu=\max _{i, k}\left|<\phi_{i}, \psi_{k}>\right|
$$


The lower the $\mu$ is, the more incoherent $\boldsymbol{\Phi}$ and $\boldsymbol{\Psi}$ are. Actually, in most WSNs or IoT, the network data can be sparsely represented on a wavelet basis $\psi$.

In compressed sensing-based framework, signal can be sampled as $\mathbf{y}=\boldsymbol{\Phi} \mathbf{x}=\boldsymbol{\Phi} \boldsymbol{\Psi} \theta$. The backbone of compressed sensing is two-fold: (1) data is compressible, only a few entries of $\theta$ have a significant amplitude; $\mathbf{x}$ is then almost entirely determined from only a few entries $\theta$; (2) measurements are incoherent: The measurement matrix $\mathbf{A}=\boldsymbol{\Phi} \boldsymbol{\Psi}$ is incoherent. In other words, the information carried by a few entries of $\theta$ will spread all over the $m$ entries of $\mathbf{y}$. Each sample $y_{k}$ is likely to contain a piece of information of each significant entry of $\mathrm{x}$.

In order to find the unique sparse solution, it is crucial to construct a measurement matrix that satisfies conditions such as null space property, restricted isometry property (RIP), and some coherence property [25].

Definition 3: For the $m$-by- $n$ measurement matrix $\boldsymbol{\Phi}$, it is said to satisfy the RIP (Restricted Isometry Property) or some coherence property of order $K$ to look for sparse solutions if there exists a $0<\delta_{k}<1$ simultaneously for all $k$-sparse signal $\mathbf{x} \in \mathbb{R}^{n}$.

$$
\left(1-\delta_{k}\right) \frac{m}{n}\|\mathbf{x}\|_{2}^{2} \leq\|\mathbf{\Phi} \mathbf{x}\|_{2}^{2} \leq\left(1+\delta_{k}\right) \frac{m}{n}\|\mathbf{x}\|_{2}^{2}
$$

In practice, we can deterministically construct the measurement matrices using random entries $\phi_{i j}$ as i.i.d realization from some probability distributions that satisfy RIP.

\section{B. Reconstruction Algorithms}

RIP can be one of the sufficient conditions for accurately reconstructing the compressed signals, which guarantees near optimal reconstruction of the solution of Eq.(2). RIP requires that the reconstruction algorithm should be able to find the sparest vector. Fortunately, this problem can be easily solved. For Eq.(2), the unknown $k$-sparse $\mathrm{x}$ can be reconstructed exactly by solving Eq.(6)

$$
\min _{\theta}\|\theta\|_{p} \quad \text { s.t. } \mathbf{y}=\mathbf{\Phi} \boldsymbol{\Psi} \theta
$$

where $\|\cdot\|_{p}=\left(\sum_{i=1}^{n}|\cdot|\right)^{1 / p}$ denotes the $\ell_{p}$-norm. Numerous results have demonstrated that $\ell_{p}(0<p \leq 1)$ satisfies RIP condition. For $\ell_{1}$, the restricted isometry constants satisfy $\delta_{k}<1$, which can guarantee the reconstruction conditions. The reconstruction of $\mathbf{x}$ can be seen as a linear or convex programming problem and many methods are available to easily solve this type of problems.

Extensive research efforts have been made to develop various sparse recovery algorithms, in which there are usually two groups of methods to perform the sparse recovery. One is convex relaxing-based recovery algorithms, such as the famous basis pursuit (BP) that aims at solving the $\ell_{1}$ minimization, Dantzig Selector, and so on; Another group of commonly used algorithms are greedy pursuit algorithms based reconstruction algorithms, such as Matched Pursuit (MP), Orthogonal Matched Pursuit (OMP), Stagewise OMP (StOMP), Compressive Sampling Matched Pursuit (CoSaMP), Subspace Pursuit (SP), and so on. Both of the convex programming-based

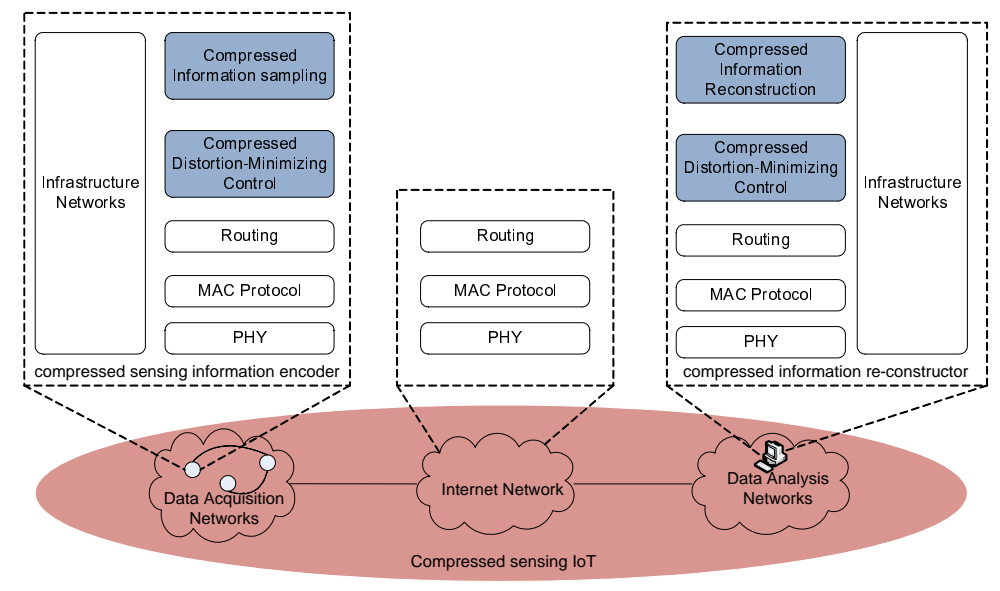

Fig. 1. Compressed sensing scheme over IoT.

and nonparametric greedy-based algorithms have advantages and disadvantages when applied to different applications. An advantage of the nonparametric greedy algorithms is that it can produce a good approximation with a small number of iterations. Meanwhile, the convex programming-based algorithms have a better reconstruction accuracy. In contrast to BP, basis pursuit de-noising (BPDN) (also called LASSO) has additional de-noising performance [27], [28], [29], [30].

\section{Noise and Reconstruction Accuracy in Compressed Sensing}

In practice, as the data has noise, LASSO is able to minimize the usual sum of square errors, with a bound on the sum of the absolute value

$$
\min _{\theta}\|\theta\|_{1} \text { s.t. } \mathbf{\Phi}^{\mathbf{T}}(\mathbf{y}-\mathbf{\Phi} \boldsymbol{\Psi} \theta) \leq \lambda_{1}
$$

Actually, Eq.(7) can also be reformatted as a penalized least squares estimate problem

$$
\underset{\theta}{\arg \min }\|\mathbf{y}-\mathbf{\Phi} \boldsymbol{\Psi} \theta\|_{2}^{2}+\lambda_{2}\|\theta\|_{\mathbf{1}}
$$

By appropriately choosing constants $\lambda_{1}$ and $\lambda_{2}$, Eq.(8) can be solved. Therefore, it is possible to accurately reconstruct the compressed signals without requiring prior knowledge when the signals are compressible over some domains. One can accurately reconstruct the sparse vector $\theta$ from compressed y using the reconstruction algorithms mentioned above and the compression rate can be defined as

$$
\rho=\frac{\|\mathbf{x}-\overline{\mathbf{x}}\|_{2}^{2}}{n}
$$

\section{CS-BASED FRAMEWORK IN WSNS AND IOT}

In this section, a compressed sensing framework for signal or data acquisition in WSNs and IoT will be introduced. It acquires a user-defined continuous packets sequence of data per interval, and after a compressed sensing-based encoding procedure the encoded packets are transmitted by wireless communications. The compressed sensing IoT (CSI) system simplifies all edge components as IoT nodes, as shown in 
Fig.1. CSI contains of three phases: (1) The design of compressed sensing information end-node (CSIE), which aims to reduce the sampling rate and the number of samples without losing the essential information; (2) The compressed data delivery scheme, compressed data is delivered to IoT networks to minimize the received data distortion and communication burden; and (3) Data reconstruction and analysis at fusion center(s). The CSI is a flexible architecture to implement a range of different information acquisition in IoT and WSNs.

\section{A. System Architecture}

The essential goal of WSNs and IoT is to accurately acquire the information about events of interest. The information acquisition networks usually consist of three core components: (1) Information sensing system, which can detect and compressively sample the signals of events; (2) Compressed sampling, the systems sample information that are preconditioned and transmitted over the networks; and (3) Reconstruction algorithms, the system accurately reconstructs the original signal from the compressed samples. Inadequate sampling may cause aliasing in signal reconstruction when the measurement matrices are not properly selected.

In contrast to conventional sensing and sampling systems, the compressed sensing can extend them to a much broader class of signals. The compressed sensing-based sampling process works by taking a small number of samples of a compressible signal on a sparse basis to reconstruct the original signals by using linear/convex optimization methods. The compressed sensing theory typically requires the projection matrix to be random, though in practice researchers have often found that the same idea can be used in other conventional sampling scenarios [2]. In this section, we summarized the signals or data that is collected by three models.

1) Node-dependent signal or data acquisition: Each node acquires i.i.d. signals. In this scenario, the compressed sensing can be used to effectively reduce the sampling rate without degenerating the reconstruction performance. A $k$-sparse signal $\mathbf{x} \in \mathbb{R}^{n}$ can be completely described by the $k$ nonzero components. $\mathrm{x}$ can be sampled with a diversifying matrix and a measurement vector $\mathbf{y}$ can be obtained. The sampling process can be described as Eq.(10)

$$
\mathbf{y}=\mathbf{A x}+\epsilon
$$

in which $\mathbf{A}$ denotes an $m$-by-n measurement matrix and $\epsilon$ is noise.

The benefits of this model are: (1) the number of samples generated by each node can be significantly reduced without losing the reconstruction accuracy; (2) it may cause the significant reduction of communications over the networks; and (3) the computation cost at nodes can be reduced.

2) Cooperative signal or data acquisition between nodes: In networks (WSN, IoT, etc.), the measurement $\mathbf{y}$ can be represented as

$$
\mathbf{y}=\left[y_{1}, \cdots, y_{m}\right]^{T}=\sum_{j=1}^{n} A_{i, j} x_{j}
$$

in which $y_{i}$ can be easily represented as a linear combination of the sparsely represented signal $x_{i}$.

Each node is able to compute $x_{j}$ by multiplying the corresponding element of matrix $A_{i, j}$, which can be constructed by choosing the entries as i.i.d realizations from some probability distribution [2]. Then randomized gossip is used to aggregate the $A_{i, j} x_{j}$ on a fusion center. By this way, $\mathbf{y}$ is available at the fusion center.

3) Consensus algorithm-based signal or data acquisition over networks: In a practical network, most nodes keep a sleeping mode based on a predefined mechanism; therefore, the topology changes over time [31]. As a result, it is necessary to take this situation into consideration for signal acquisition or data collection.

Consider a network with $n$ nodes at location $\left\{p_{i}\right\}$ ( $i=$ $1, \ldots, n)$ is monitoring multiple events, assume that $\mathcal{N}_{a}(t)$ nodes are in active mode and $\mathcal{N}_{s}(t)$ nodes are in sleep mode at time $t$. Let $x_{i}$ denote the source value at $p_{i}, i \in n$. Then measurement $y_{i}$ of node $i$ can be represented as

$$
y_{i}=\sum_{j \in N} A_{j, i} x_{j}+\epsilon_{i}
$$

in which $A_{i, j}=A_{j, i}$ is the influence of this event on sensor point $p_{i}$, and $\epsilon_{i}$ is the random measurement noise of zero mean. Here $\mathbf{x}$ is sparse and $A_{i, j}$ can be learned during the network deployment stage.

Assume that the influence $A_{j i}=0$, if the distance from $j$ to $i$ is larger than the communication range. Then the measurement $y_{i}$ becomes $y_{i}=x_{i}+\sum_{j \in n} A_{j, i} x_{j}+\epsilon_{i}$, furthermore, for the active nodes in the network, we have

$$
\mathbf{y}_{a}=\boldsymbol{\Phi} \mathbf{A} \mathbf{x}+\epsilon_{a}
$$

where $\mathbf{A}$ is the $n \times n$ matrix with $(i, j)$-th element being $\left(A_{i, j}\right)$, $\Phi$ is the $m \times n$ measurement matrix that selects the $m$ rows of $A$ corresponding to the active sensors, and $\mathbf{y}_{a}$ and $\epsilon_{a}$ are the $m \times 1$ measurement vector and noise vector, respectively.

In compressed sensing theory, we aim to recover the $n \times 1$ sparse signal vector $\mathbf{x}$ from $m$ measurements. This can be solved as an optimization problem

$$
\min _{\mathbf{x}}\left\|\mathbf{A x}-\mathbf{b}_{\mathbf{a}}\right\|_{2}^{2}+\lambda_{2}\|\mathbf{x}\|_{1} \text { s.t. } \mathbf{x} \geq \mathbf{0}
$$

Let $\mathcal{N}_{i}$ denote the neighboring nodes of $i$. Assume that each active sensor $i$ holds the signal $x_{i}$ at its own location as well as the signals $x_{k}$ occurring at its inactive neighboring node $\forall k \in \mathcal{N}_{a} \cup \mathcal{N}_{i}$. This means node $i$ keeps its measurement $x_{i}$ and $\left\{x_{k}\right\}_{k \in \mathcal{N}_{i}}$. Then Eq.(14) can be reformulated as

$$
\min \sum_{i \in \mathcal{N}_{a}}\left(y_{i}-x_{i}^{(i)}-\sum_{k \in \mathcal{N}_{s} \cup \mathcal{N}_{i}} A_{k, i} x_{k}^{(i)}-\sum_{j \in \mathcal{N}_{a}} A_{k, i} x_{j}^{(j)}\right)^{2}
$$

$$
\begin{aligned}
\text { s.t. } x_{i}^{(i)} & \geq 0, \forall i \in \mathcal{N}_{a}, \\
x_{k}^{(i)} & \geq 0, \forall k \in \mathcal{N}_{s} \cup \mathcal{N}_{i}
\end{aligned}
$$

Eq.(15) can be reformulated as a separable convex program, which can be solved with a consensus algorithm by using the alternating direction method of multipliers. 


\section{B. Sparse Representation}

The CSIE samples the original information based on compressed sensing theory and then deliver the samples through CSI. The sampling rate (or the number of samples) is determined in this process, while the measurement matrix is pre-selected and shared between the sender and receiver as described in Section II.A.

CSIE has two advantages: (1) it runs on low complexity and can be used over thin-node of IoT; and (2) it takes the advantage of the temporal correlation between continuous data matrices. CSIE is able to effectively sample the compressible signal $\mathbf{x}$ on a certain basis. However, a few promising schemes are available for the design of the CS-based information encoder to sparsify/compress bases for information or data. Since an information system (e.g., wireless sensor network) consists of many data sources that are able to monitor the information related to a certain spatially varying phenomenon, if the nodes are deployed in a random manner such that they cover uniformly a given surface, then sparsifying transformation may be readily borrowed from traditional signal processing. In this case, many well-developed tools such as discretecosine-transform (DCT), discrete-Fourier-transform (DFT) or discrete-wavelet-transform (DWT) may be used to de-correlate and sparsify the sensor data [4].

Fig.2 illustrates an example of compressed sensing-based spatially correlated data acquisition network, where DWT is used for sparsification. Actually, the non-sparse raw data in Fig.2(a) can easily be sparsely represented over a wavelet basis as shown in Fig.2(b).

In IoT, remote data collection involves specific collections that often provide redundant data which cannot be accounted for by a standard compression technique. In a general framework, let us consider that $N$ observations of the same monitoring area are available: $\left\{y_{i}\right\}(i=1, \cdots, N)$ such that

$$
y_{i}=A_{\Lambda_{i}} x+n_{i}
$$

where $\left\{A_{\Lambda_{i}}\right\}(i=1, \cdots, N)$ are $N$ independent random submatrices of $\Phi$ with $\operatorname{Card}\left(\Lambda_{i}\right)=M$. It is clear that $x$ can be reconstructed from the $N$ compressed observations $\left\{y_{i}\right\}_{i=1, \ldots, N}$. According to Eq.(5), we propose a substitution decomposition solution with the following

$$
\min _{\theta}\|\theta\|_{1} \quad \text { s.t. } \sum_{i=1}^{N}\left\|\mathbf{y}_{\mathbf{i}}-\mathbf{A}_{i, j} \theta\right\|_{2}^{2} \leq \epsilon
$$

It can be further recast in the following Lagrangian form

$$
\underset{\theta}{\arg \min } \sum_{i=1}^{N}\left\|\mathbf{y}_{\mathbf{i}}-\mathbf{A}_{i, j} \theta\right\|_{2}^{2}+\lambda_{2}\|\theta\|_{1}
$$

For a sensor network with changing topology that the data is made of $N$ readings $\left\{x_{i}\right\}_{i=1, \cdots, N}$ such that each reading $x_{i}$ is a noiseless observation of the same sensing area $x$, each observation is compressed using compressed sensing such that $\rho=M / t$. Compression is made by solving the problem in

$$
\min _{\theta} \sum_{i=1}^{N}\left\|y_{i}-\phi_{i} \Psi \theta\right\|_{2}^{2}+\lambda_{2}\|\theta\|_{1}
$$

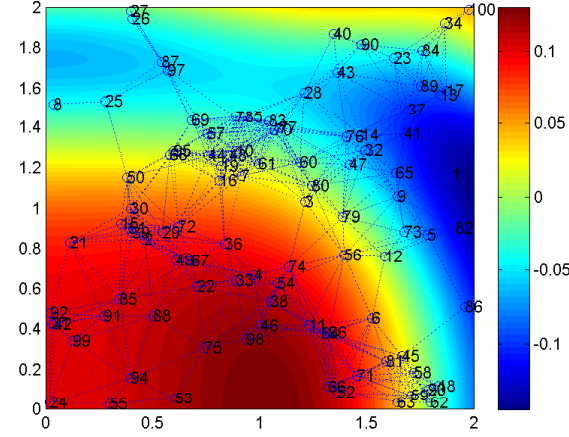

(a) Monitoring scenario

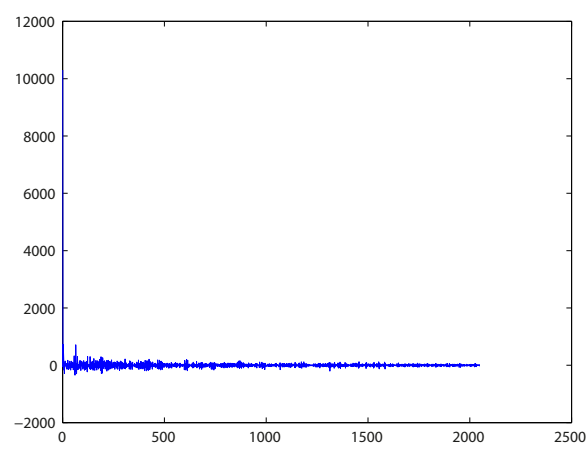

(b) Sparse representation of monitoring data

Fig. 2. Illustration of the compressibility of network.

in which $\lambda_{2}$ is a regularization parameter, which is a trade-off between the sparse representation of signals and reconstruction accuracy.

\section{Noise Model, Communication Load, and Recovery Accu- racy}

This subsection will discuss the noise model in compressed sensing. For compressed problem the measurement noise can be modeled as

$$
\mathbf{y}=\mathbf{A x}+\epsilon
$$

where $\mathbf{y} \in \mathbb{R}^{n}$ is the measurement, $\mathbf{A} \in \mathbb{R}^{m \times n}$ is the measurement matrix, and $\epsilon$ is assumed to be a Gaussian random vector with i.i.d. components. Let $I_{m}$ is an identity matrix of size $m$, for the normalized measurement matrix $\mathbf{A}$ $=\left[A_{i, j}\right]$, each component here is assumed to be distributed as $A_{i, j} \sim \mathcal{N}(0,1 / m), i=1, \ldots, m$, and $j=1,2, \ldots, n$.

Similarly, the input noise model can be given by

$$
\mathbf{y}=\mathbf{A}(\mathbf{x}+\epsilon)
$$

where $\epsilon \sim \mathcal{N}\left(0, I_{n}\right)$ is a Gaussian random vector with i.i.d components.

In multihop networks (such as WSNs, IoT, and so on), the random projection of sensing data can be computed and delivered to every subset of nodes using a gossip or consensus scheme, or they might be delivered to FC(s) using clustering and aggregation techniques [4]. In some ad hoc networks, explicit routing information is difficult to be obtained and 
maintained, and each node jointly contributes its measurement to the FC.

In WSNs or IoT, the nodes transmit packets asynchronously, which may cause packets collision at the gateway or FC [32]. If one or more bits in a packet are in error, then a packet will be in error, and the probability of a packet in error can be modeled as [32], [33]

$$
P_{E}=1-\left(1-P_{e}\right)^{L}
$$

where $P_{e}$ denotes the probability of bit error and $L$ denotes the number of bits of per packet which is related to the $S N R$, e.g., $P_{e}=1 / 2 e^{-S N R}$ for DPSK. Actually, in IoT, Poisson process can be used to model the useful packet generation, transmission, and arrival at each node. For an IoT network (or WSN) with $N$ nodes, we assume that the arrival of useful packets follows a Poisson process with an effective average arrival rate at $\mathrm{FC}$

$$
\sigma=\frac{N\left(1-e^{\eta T}\right) e^{-2 N \eta T_{p}}\left(1-P_{E}\right)}{T}
$$

where $\eta$ denotes the average packet-generation-rate at a node, $T$ denotes a data frame collection interval, and $T_{p}$ denotes the packet duration [32], [34]. Then, the number of useful packets for the reconstruction algorithm can be described as

$$
\operatorname{Prob}\{K(\eta, T)=k\}=P_{K}(k ; \eta, T)=\frac{(\eta T)^{k}}{k !} e^{-\sigma T}
$$

In IoT and WSNs, communication burden is a major concern for decentralized algorithm design. In the proposed approach, nodes need to exchange intermediate information in each iteration, which can be done via local broadcasting. With compressed sensing, communication load can be reduced from two sides: (1) The nodes just need to exchange intermediate information in each iteration via local broadcasting; (2) Only the data needs to be transmitted is compressed since only a small part of measurements are needed without the loss of essential information.

In practice, it is necessary to accurately reconstruct the compressed data, and the recovery accuracy shall be assessed based on the network goal. The accuracy of recovery is determined by the recovery algorithm, the communication range, and the collected data that shall contain adequate information.

\section{Adaptive Cluster Sparse Representation and Recovery Al- gorithm}

The sensing data in most applications in IoT or WSNs often exhibits a certain degree of correlation, therefore there is large space to compress sensing reports to reduce transmissions. In this section we will propose an adaptive cluster sparse recovery algorithm by taking both the sparsity and correlation of data into consideration. Actually the correlation of data is always ignored by most pre-existing CS reconstruction algorithms. At first, we introduce a definition for adaptive cluster sparsity as

Definition 4: Cluster-sparse. If a signal $\mathrm{x}$ can be accurately represented with $k \ll n$ nonzero components (or over some transform domains), these $k$ nonzero elements can be clustered into $c \in\{1, \cdots, k\}$ clusters.
If the size of all clusters is 1 , then the $c$ cluster-sparse representation of signal is exactly the traditional $k$-sparse representation. The 'adaptive' means that the number of clusters $c$ may be unknown and it changes dynamically. It is obtained according to the geodesic distance between two neighboring samples as described in Algorithm 1. The prior knowledge about signal is that $\mathbf{x}$ is adaptively cluster-sparse, which is not necessary to span all $k$-dimensional subspace in the union $\Omega_{k}$ that is used in conventional compressed sensing. Actually, the cluster-sparse representation can significantly decrease the degree of signals than in $k$-sparse case. By doing so, the minimal measurement number $m$ can be decreased for robust signal reconstruction [2], [21].

It is clear that cluster-sparse scheme can effectively reduce the number of measurements required for robust signal reconstruction to $m=O\left(k+c \cdot \log \left(\frac{n}{c}\right)\right)$. Compared with the number of measurements $m=O\left(k \log \left(\frac{n}{k}\right)\right)$ that is acquired by conventional CS recovery algorithm, it significantly improves the compressibility of signal.

In this section we will propose an adaptive cluster sparse reconstruction algorithm (ACSRA), which can better balance the scales of the components and computation complexity among existing algorithms such as CoSaMP and dynamic group sparse (DGS) [35]. ACSRA includes following five main steps in each iteration:

1) Estimate the residual of each iteration;

2) Compute the best clusters $C_{k, c}$ support set of the errors (index set);

3) Merge the strongest support set;

4) Reconstruct the signals according to the given support sets;

5) Prune $x$ and computer residual for the next round.

Different from point-sparse approximation algorithms, in ACSRA we prune the reconstructed signal and residue based on a cluster sparse approximation. Here we only need to search the sparse cluster subspace $C_{k, c}$, which causes the reduction of measurements for stable signal recovery.

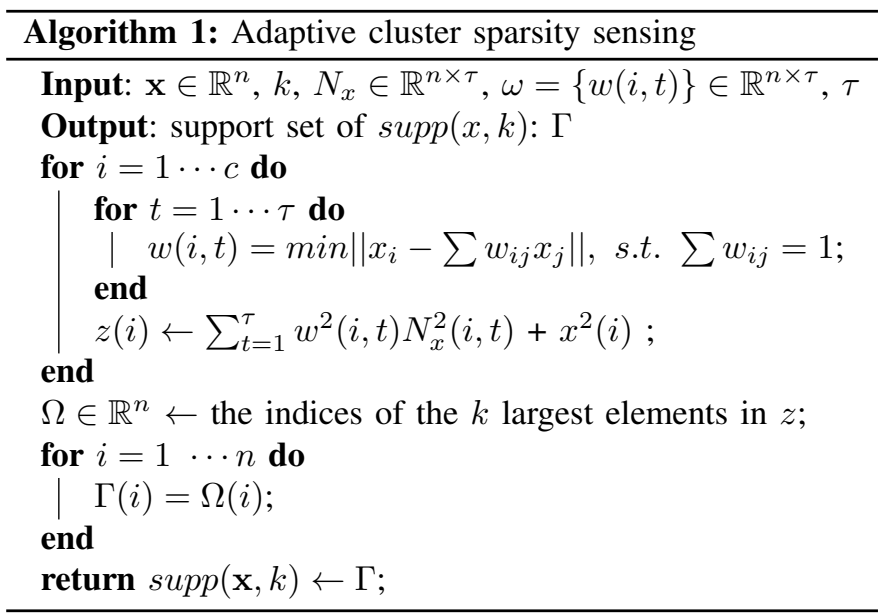

Details of the ACSRA cluster-sparse sensing scheme can be found in Algorithm 1, where two parameters $\tau$ and $w$ are applied to tune the number of neighbours and its weights. In the cluster-sparse scheme, $\tau$ can adjust the number of 
neighbours in sparse data and $w$ can balance the cluster priorknowledge and the sparsity of signal. Here $w$ is the local combination weights vector that is determined by solving a least squares problem. In Algorithm 1, the weights can be obtained by solving a linear local embedding problem based on the pairwise distance between two data points.

In practice, it is difficult to obtain the sparsity number $k$ in signal recovery. Fortunately, in many practical applications, the sparsity number may range from $k_{\min }$ to $k_{\max }$ [35]. In continuously signal acquisition, the $\left[k_{\min }, k_{\max }\right]$ can be estimated depending on the sparsity of signal. The step size can be the acquisition resolution. Let $\triangle k$ denote the step size of sparsity number and the reconstruction of signal or data can be obtained by Algorithm 2, in which $\epsilon$ bounds the amount of noise in the signals.

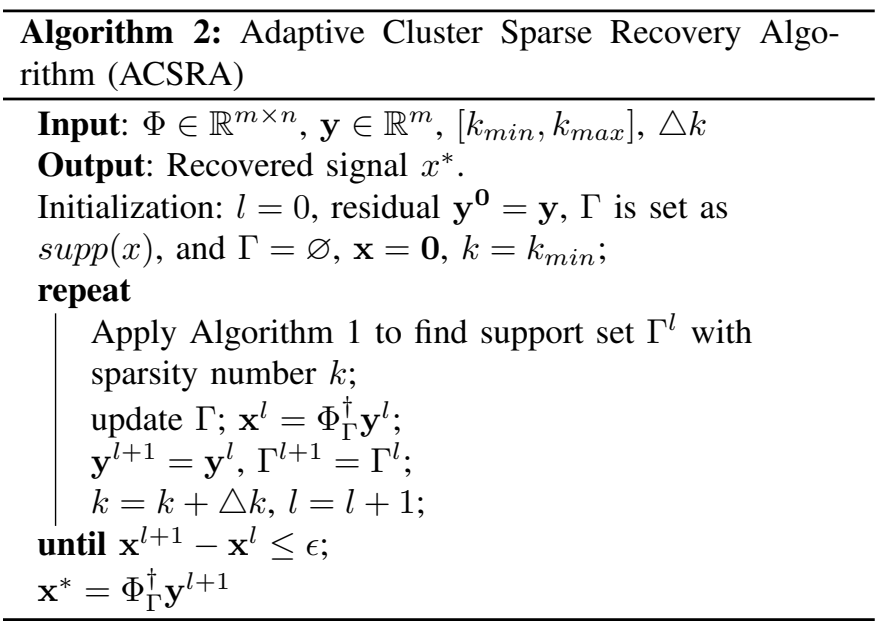

\section{PERFormance EVAluation}

This section provides extensive simulation results to demonstrate the effectiveness of the proposed sparse signal recovery algorithm in WSNs and IoT. At first, a small IoT network is built to acquire ECG signals, which can demonstrate the basic properties of the proposed algorithm. Then, a large IoT network is simulated to demonstrate the scalability of the decentralized algorithm.

\section{A. Nodes-Dependent Signal Acquisition}

For node-dependent signal or data acquisition in wireless sensor networks or IoT, we assume each node in the network is able to acquire an ECG signal, which is very common in healthcare systems. In this simulation experiment, as signal source, the dataset is obtained from the archives at the University of California (http://www.cs.ucr.edu/eamonn/discords/), we consider seven real ECG signals datasets (SAX) from different movements of subjects.

We compare the performance of the reconstruction accuracy and reconstruction speed for different compressed recovery algorithms. All of them significantly decrease the volume of sampling comparing with conventional Nyquist sampling scheme. Table.I also shows the recovery error and the CPU times that used by the proposed algorithm for different measurements and $K$-sparse ECG signals. Table $I$ shows that the recovery accuracy of seven ECG signals in datasets, in which the 'Recovery Error' can be calculated by $\sqrt{\sum_{i=1}^{N}\left(\hat{x}_{i}-x_{i}\right)^{2} / N}$.

It is clear that when $k$ is properly selected, the signals can be recovered perfectly, however, the number of samples is much smaller than that of Nyquist. For real time monitoring over WSNs or IoT, this scheme can significantly reduce the power consumption and communication loads over the whole networks.

TABLE I

DATASETS EXPERIMENTS PARAMETER AND RECOVER PERFORMANCE

\begin{tabular}{ccccc}
\hline No. & ECG Datasets & Size $(N)$ & Recovery Error & CPU Time $(\mathrm{s})$ \\
\hline 1 & chfdb1 & 3750 & 0.0360 & 2.22690 \\
2 & chfdb2 & 3750 & 0.0280 & 1.50751 \\
3 & ltstdb1 & 3750 & 0.1066 & 1.30932 \\
4 & ltstdb2 & 3750 & 0.0691 & 1.48217 \\
5 & mitdb & 5400 & 0.0426 & 3.69623 \\
6 & stdb & 5400 & 0.1193 & 2.10079 \\
7 & xmitdb & 5400 & 0.0139 & 3.42036 \\
\hline
\end{tabular}

Notice that the ECG signals themselves are not sparse in the time-domain. We need to transform the ECG signals into other sparse domains, such as the wavelet domain. We contracted the signal as a $1 \times 4096$ vector. The size of the observation matrix for the nearly perfect reconstruction should be at least larger than $M \times 4096$ where $M$ is calculated by $M=O(k+$ $c \cdot \log (N / c))(k$ is the sparse level, in our experiment $k=$ $128, c$ denotes the number of clusters. The length of signal is $2048)$, which is a significant improvement than $m=O(k+$ $k \cdot \log (n / k))$. The latter is widely used in existing compressed sensing recovery algorithms. In our experiments the minimal $M$ is 256 . Actually we relaxed this minimal value to 384 to obtain more reliable results. It means that only $18.75 \%$ of the original data needs to be transmitted over WSN, with which the original signal can be perfectly reconstructed. Under this situation, the communication load over WSN can be decreased by $81.25 \%$.

For comparison's sake, we tested three commonly used recovery algorithms: GPSR, LASSO, OMP, and proposed ACSAR by simulations on the ECG signal ( $\left.c h f d b \_c h f-1\right)$ with length of 2048. The GPSR is proposed for bound-constrained optimization to find the sparse solution, which shows a fast and accurate performance for data with group/cluster sparsity structure, such as image or continuous signals. LASSO is the most well-known $\ell_{1}$ minimization in CS, which performs well in a broad range of circumstances. The performance of OMP has proven to be effective in solving sparse solution to reverse problem arising in overcomplete representation.

Fig.3(a) and 3(b) show the original ECG signal (chfdb_chf - 1) with length of 2048 and its sparse representation of ECG signal, which includes 1974 entries less than 0.5. Fig.4(a), (b), and (c) compare the reconstructed ECG signals by the algorithms GPSR, LASSO, and OMP that are widely used in compressed sensing recovery; Fig.4(d) gives the reconstructed ECG signal by proposed ACSAR. The average recovery errors shown in Fig.4(a), (b), and (c) are $0.3883,0.2634$ and 0.3239 , respectively. The average recovery error of proposed ACSAR shown in Fig.4(d) is 


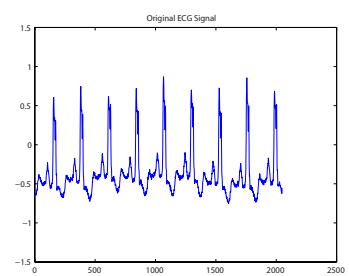

(a) Original ECG signal

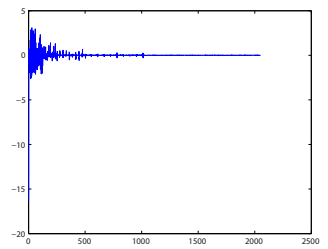

(b) Sparse representation of ECG
Fig. 3. ECG signal and its' sparse representation (chfdb_chf-1)

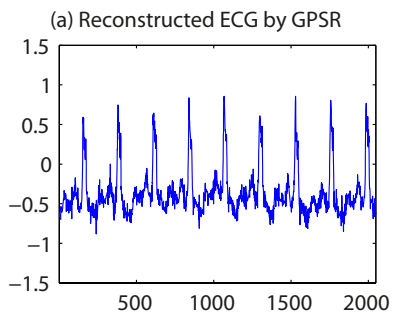

(b) Reconstructed ECG by LASSO
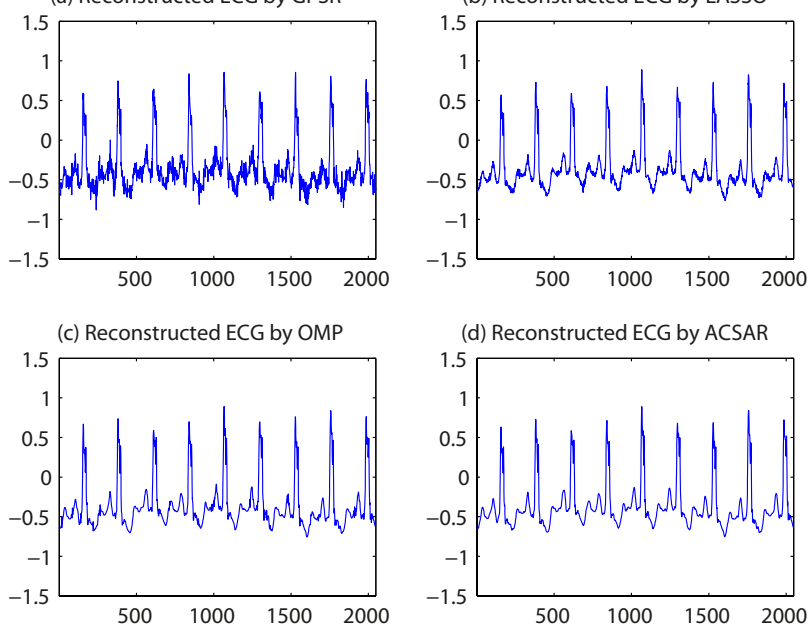

(d) Reconstructed ECG by ACSAR

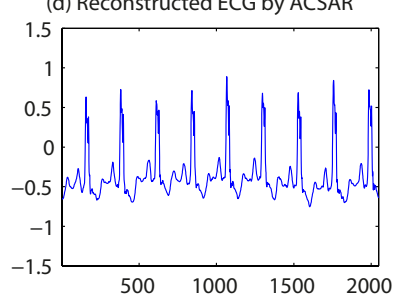

Fig. 4. ECG original signal (chfdb_chf -1$)$ and reconstructed signals with GPSR, LASSO, OMP and ACSAR $(k=74, M=512)$.

0.0878. It can be seen that proposed recovery algorithm can achieve more accurate performance than GPSR, LASSO, and OMP when the same measurements are used.

\section{B. Cooperative Signal and Data Acquisition}

To visually illustrate the cooperative signal acquisition process, we employ a WSN to sense a $5 \times 5$ unites field as shown in Fig.5(a) and 5(b). Each node acquires a measurement that may, for instance, be the humidity or temperature. Actually, the Fourier transform of the measurements shows a sparse representation with sparsity $k=16$. For a $1000 s$ interval, a packet size $L=1000$ bits, and a packet duration $T_{p}=0.5 \mathrm{~s}$. Packets are sent to the fusion center at a bit rate of $5 \mathrm{kbps}$ and a packet error probability $P_{E}=0.1$.

A number of sensors monitor the signal and periodically report the readings by wireless communications to the FC. Each node senses the environment with a CS framework and transmits the readings to the $\mathrm{FC}$. When $\mathrm{FC}$ receives the compressed projections of readings from the nodes, the signal at each node can be reconstructed by using the proposed ACSRA algorithm. Employing the proposed ACSRA algorithm, a compressible rate of $26 \%$ can be obtained, and the reconstructed map can be available in Fig.5(b) in which the original signals can be reconstructed with high probability as great as $95 \%$.

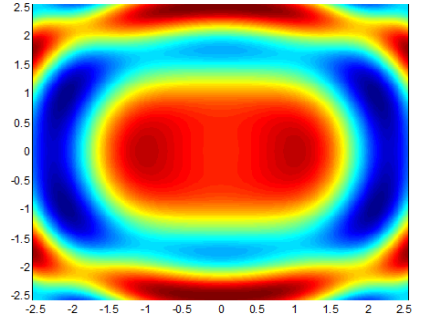

(a) Original map of the sensing area.

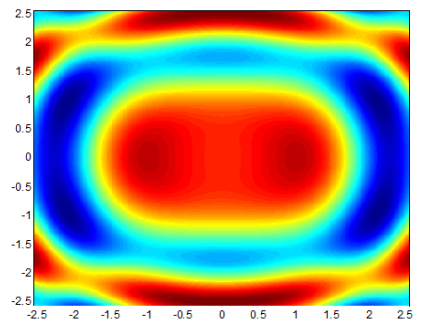

(b) Reconstructed map of the sensing area.

Fig. 5. The original map and reconstructed map with ACSRA algorithm

\section{CONClusion}

In this paper, we first proposed a compressed sensing framework for WSNs and IoT and introduced how the framework could be utilized to reconstruct the sparse or compressible information data into a variety of information systems involving with WSNs and IoT. The compressed sensing-based framework provides a promising approach for compressible signal and data in information systems by employing a priori data sparsity information, which makes it an effective new information and data gathering paradigm in networks and information systems.

This work has shown that compressed sensing can be a powerful data acquisition tool for saving energy and communication resources in networks and information systems. It further strengthens the connection between information theory and compressed sensing. As part of our ongoing work, we are investigating the best achievable compressed sensing reconstruction schemes over information systems and networks.

\section{REFERENCES}

[1] Lida Xu, "Enterprise Systems: State-of-the-Art and Future Trends", IEEE Transactions on Industrial Informatics, vol.7, no.4, pp.630-640, September 2011.

[2] Jun Zheng David Simplot-Ryl Chatschik Bisdikian Hussein T. Mouftah, "The internet of things", IEEE Communications Magazine, vol.49, no.11, pp.30-31, November 2011.

[3] Luigi Palopoli, Roberto Passerone, and Tizar Rizano, "Scalable Offline Optimization of Industrial Wireless Sensor Networks", IEEE Transactions on Industrial Informatics, vol.7, no.2, pp.328-329, May 2011.

[4] Jarvis Haupt, Waheed U.Bajwa, Michael Rabbat, and Robert Nowak, "Compressed Sensing for Networked Data: A different approach to decentralized compression", IEEE Signal Proceesing Magazine, vol.25, no.2, pp.92-101, March 2008.

[5] Alphan Ulusoy, Ozgur Gurbuz, and Ahmet Onat, "Wireless ModelBased Predictive Networked Control System Over Cooperative Wireless Network", IEEE Transactions on Industrial Informatics, vol.7, no.1, pp.41-51, February 2011.

[6] M. Jongerden, A. Mereacre, H. Bohnenkamp, B. Haverkort and J.P. Katoen, "Computing Optimal Schedules of Battery Usage in Embedded Systems", IEEE Transactions on Industrial Informatics, vol.6, no.3, pp.276-286, August 2010.

[7] Konstantinos Kakousis, Nearchos Paspallis, and George Angelos Papadopoulos, "A survey of software adaptation in mobile and ubiquitous computing", Enterprise Information Systems, vol.4, no.4, November 2010.

[8] Jerome Bobin, Jean-luc Starck, and Roland Ottensamer, "Compressed Sensing in Astronomy", IEEE Journal of Selected topics in Signal Processing, vol.2, no.5, pp.718-727, October 2008.

[9] Shancang Li, Lida Xu, Xinheng Wang, and Jue Wang, "Integration of hybrid wireless networks in cloud services oriented enterprise information systems", Enterprise Information Systems, vol.6, no.2, pp.165-187, January 2012. 
[10] Vehbi C. Gungor, Dilan Sahin, Taskin Kocak, Salih Ergut, Concettina Buccella, Carlo Cecati, and Gerhard P. Hancke, "Smart Grid Technologies: Communication Technologies and Standards", IEEE Transactions on Industrial Informatics, vol.7, no.4, pp.529-539, November 2011.

[11] Chuan Fu, Guoqing Zhang, Jing Yang, and Xiaona Liu, "Study on the contract characteristics of Internet architecture", Enterprise Information Systems, vol.5, no.4, pp.495-513, November 2011.

[12] Sameer Kumar, Brooke Kadow, and Melissa Lamkin, "Challenges with the introduction of radio-frequency identification systems into a manufacturer's supply chain for a pilot study", Enterprise Information Systems, vol.5, no.2, pp.235-253, May 2011.

[13] Hossein Mamaghanian, Nadia Khaled, David Atienza, and Pierre Vandergheynst, "Compressed Sensing for Real-Time Energy-Efficient ECG Compression on Wireless Body Sensor Nodes", IEEE Transactions on Biomedical Engineering, vol.58, no.9, September 2011.

[14] Maxim Raginsky, Sina Jafarpour, Zachary T.Harmany, and Robert Calderbank, "Performance Bounds for Expander-Based Compressed Sensing in Poisson Noise", IEEE Transactions on Signal Processing, vol.59, no.9, September 2011

[15] Fatemeh Fazel, Maryam Fazel and Milica Stojanovic, "Random Access Compressed Sensing for Energy-Efficient Underwater Sensor Networks", IEEE Journal of Selected Areas in Communications, vol.29, no.8, pp.1660-1670, September 2011.

[16] Scott Pudlewski, Tommaso Melodia, and Arvind Prasanna, "Compressed-Sensing-Enabled Video Streaming for Wireless Multimedia Sensor Networks", IEEE Transactions on Mobile Computing, vol.11, no.6, pp.1060-1062, June 2012.

[17] Hossein Mamaghanian, Nadia Khaled, David Atienza,and Pierre Vandergheynst, "Compressed Sensing for Real-Time Energy-Efficient ECG Compression on Wireless Body Sensor Nodes", IEEE Transactions on Biomedical Engineering, vol.58, no.9, pp.2456-2466, September 2011.

[18] Lida Xu, "Information Architecture for Supply Chain Quality Management", International Journal of Production Research, vol.49, no.1, pp.183-198, January 2011.

[19] Scott Pudlewski, Arvind Prasanna, and Tommaso Melodia, "Compressed-Sensing-Enabled Video Streaming for Wireless Multimedia Sensor Networks", IEEE transactions on Moble Computing, 2011.

[20] Carlo Caione, Davide Brunelli, and Luca Benini, "Distributed Compressive Sampling for Lifetime Optimization in Dense Wireless Sensor Networks", IEEE Transactions on Industrial Informatics, vol.8, no.1, pp.30-40, February 2012.

[21] Richard G. Baraniuk, Volkan Cevher, Marco F. Duarte, and Chinmay Hegde, "Model-Based Compressive Sensing", IEEE Transactions on Information Theory, vol.56, no.4, pp.1982-2001, April 2010.

[22] Emmanuel J. Candes and Michael B. Wakin"An Introduction To Compressive Sampling”, IEEE Signal Processing Magazine, vol.25, no.2, pp.21-30, March 2008.

[23] Xi Chen, Zhuizhuan Yu, Hoyos S., Sadler B.M., Silva-Martinez J., "A Sub-Nyquist Rate Sampling Receiver Exploiting Compressive Sensing", IEEE Transactions on Circuits and Systems I: Regular Papers, vol.58, no.3, pp.507-520, March 2011

[24] Chong Luo, Feng W, Jun Sun, and Changwen Chen, "Efficient Measurement Generation and Pervasive Sparsity for Compressive Data Gathering", IEEE Transactions on Wireless Communications, vol.9, no.12, pp.3728-3738, October 2010.

[25] Richard Baraniuk, Mark Davenport, Ronald DeVore, and Michael Wakin, "A Simple Proof of the Restricted Isometry Property for Random Matrices", Constructive Approximation, vol.28, no.3, pp.253-263, December 2008.

[26] Lihi Zelnik-Manor, Kevin Rosenblum, and Yonina C. Eldar, "Sensing Matrix Optimization for Block-Sparse Decoding", IEEE Transactions on Signal Processing, vol.59, no.9, pp.4300-4312, September 2011.

[27] Michael A. Lexa, Mike E. Davies, and John S. Thompson, "Reconciling Compressive Sampling Systems for Spectrally Sparse Continuous-Time Signals", IEEE Transactions on Signal Processing, vol.60, no.1, pp.155171, January 2012.

[28] Zhaorui Liu, A. Y. Elezzabi, and H. Vicky Zhao, "Maximum Frame Rate Video Acquisition Using Adaptive Compressed Sensing", IEEE Transactions on Circuits and Systems for Video Technology, vol.21, no.11, pp.1704-1718, November 2011.

[29] T. Blumensath and M. Davies, "Sampling theorems for signals from the union of finite-dimensional linear subspaces", IEEE Transactions on Information Theory, vol.55, no.4, pp.1872-2882, 2009.

[30] Arian Maleki and David L. Donoho, "Optimally Tuned Iterative Reconstruction Algorithms for Compressed Sensing", IEEE Journal of Selected Topics in Signal Processing, vol.4, no.2, pp.330-341, April 2010.
[31] Qing Ling and Zhi Tian, "Decentralized Sparse Signal Recovery for Compressive Sleeping Wireless Sensor Networks", IEEE Transactions on Signal Processing, vol.58, no.7, pp.3816-3828, July 2010.

[32] Fatemeh Fazel, Maryam Fazel and Milica Stojanovic, "Random Access Compressed Sensing for Energy-Efficient Underwater Sensor Networks", IEEE Journal of Selected Areas in Communications, vol.29, no.8, pp.1660-1670, September 2011.

[33] David Wang, Jun Liu, and Rajagopalan Srinivasan, "Data-Driven Soft Sensor Approach for Quality Prediction in a Refining Process", IEEE Transactions on Industrial Informatics, vol.6, no.1, pp.11-17, February 2010.

[34] Sooyeon Shin, Taekyoung Kwon, Gil-Yong Jo, Youngman Park, H. Rhy, "An Experimental Study of Hierarchical Intrusion Detection for Wireless Industrial Sensor Networks", IEEE Transactions on Industrial Informatics, vol.6, no.4, pp.744-757, November 2010.

[35] Shaoting Zhang, Junzhou Huang, Hongsheng Li, and Dimitris N. Metaxas, "Automatic image annotation using group sparsity", IEEE Transactions on Systems, Man, and Cybernetics, Part B: Cybernetics, vol.42, no.1, pp.1-12, January 2012. 